\title{
Hypoxia is no hype: Perspectives across Phylogeny, Stem Cell differentiation \& Geochemistry
}

Influence of atmospheric gases in the eco systems on the habituating living organisms, an area of focus of the geochemists and astro-biologists, have been known for eons. Though the modus operandi of the implication varies between unicellular and multi-system centered organisms up in the ladder of phylogeny, their significance though known, the intricacies to the fullest extent have not been thoroughly understood to enable us to exploit them to our best in bringing solutions to address various problems.

In this issue, Ito et al have reported a simple hypoxic culture system $^{[1]}$ with a built-in deoxidizing agent capable of promoting stem cell proliferation, repressing cell senescence without aggravating the stem cell viability. Studying hypoxia gains significance because oxygen exerts a profound influence on life on earth. Oxygen requirements differ across cells, tissues, organisms and phylogeny. The primitive life forms on earth which formed nearly four billion years ago were able to thrive without oxygen, which became a life influencing factor only two billion years ago during when its levels in the atmosphere were actually toxic to the organisms which evolved ways to detoxify them and over time these organisms became dependant on oxygen ${ }^{[2]}$. Among cellular processes, the low energy supply during a hypoxic metabolic state allows only DNA replication or basic cell reproduction functions which are essential for a cell's survival suppressing the specialised functions like differentiation ${ }^{[2]}$. Thus, stemness is preserved more efficiently under hypoxia and it is suggested that in vitro stem cells should ideally be maintained at oxygen concentrations lower than $1 \%$ and approaching zero which recapitulate the atmospheric oxygen concentrations that existed on earth 2-3 billion years ago, as stem cells are believed to reflect an early evolutionary stage compared to other cells ${ }^{[2]}$. Hypoxia has shown to promote stemness in various kinds of stem cells including the embryonic, hematopoietic, mesenchymal and neural stem cells $^{[3]}$. Hypoxia also becomes important in cancer biology as it promotes the self-renewal of the cancer initiating cells or cancer stem cells ${ }^{[4]}$.

In vitro models of meticulous documentation of the dynamic gaseous inter-cellular and intra-cellular environments similar to the one reported by lto et al if evolved can be put in use for future in vivo manipulation of parameters like hypoxia to have a better understanding of its influence over cellular differentiation and lineage specification.

\section{References}

1. Ito A, Aoyama T, Yoshizawa M, Nagai M, Tajino J, Yamaguchi S, lijima $H$, Zhang $X$, Kuroki $H$. The effects of short-term hypoxia on human mesenchymal stem cell proliferation, viability and p16INK4A mRNA expression: Investigation using a simple hypoxic culture system with a deoxidizing agent. J Stem Cells Regen Med. 2015; 11(1):25-31.

2. Ivanovic Z. Hypoxia or in situ normoxia: The stem cell paradigm. J Cell Physiol. 2009 May; 219(2):271-5.

3. Mohyeldin A, Garzón-Muvdi T, Quiñones-Hinojosa A. Oxygen in stem cell biology: a critical component of the stem cell niche. Cell Stem Cell. 2010 Aug 6;7 (2):150 61.

4. Li Z, Rich JN. Hypoxia and hypoxia inducible factors in cancer stem cell maintenance. Curr Top Microbiol Immunol. 2010; 345:21-30. 